\title{
Therapeutic Results of Transcutaneous Electrical Nerve Stimulation in Post Laminectomy Syndrome
}

\author{
Ahmad Shokrzadeh \\ Assistant professor of Anesthesiology, Rajaie Hospital \\ Qazvin University of Medical Sciences, Qazvin, Iran \\ Tel: 98-281-333-5800 E-mail: ashokrzadeh@qums.ac.ir \\ Afsoun Seddighi (Corresponding author) \\ Assistant professor of Neurosurgery, Rajaie Hospital \\ Qazvin University of Medical Sciences, Qazvin, Iran \\ Tel: 98-281-333-5800Ｅ-mail: aseddighi@qums.ac.ir
}

\author{
Amir Saied Seddighi \\ Neurosurgeon, Assistant Professor of Neurosurgery, Shohada Tajrish Hospital \\ Beheshti University of Medical Sciences, Tehran, Iran \\ Member of Neurofunctional Research Center of Beheshti University \\ Tel: 98-218-826-5188Ｅ-mail: a_sedighi@sbmu.ac.ir
}

\begin{abstract}
Objectives \& Background: Post-laminectomy syndrome specifically refers to pain associated with symptoms not relieved following laminectomy. This entity is a very disabling and hard to manage problem in patients undergoing spinal procedures. Transcutaneous electrical nerve stimulation has been introduced as a non invasive modality for pain control. This study was aimed to determine the effectiveness of TENS in the management of postlaminectomy syndrome. No similar study has been performed in our country yet.

Materials and Methods: In this study 35 patients among 85 serial cases with post laminectomy syndrome from 2008-2009 were treated by transcutaneous stimulation. Age, sex, duration and type of symptoms, topographical locations, radiological and the results, for pain control were analyzed. Pain degree determined before stimulation and then 2 hours and 48 hours afterwards using visual analog scale.

Results: This study is based on the data from 35 serial patients visited in the senior author's clinic. $60 \%$ of the patients were male. The average age of our patients was 49.5 years. The most common location was L4-5 interspace (40\%). Average pain score before stimulation was 7 and declined to the mean 5 after 2 hours and to 4 after 48 hours.

Conclusions: According to our study the successful results of transcutaneous stimulation occurred in $17.14 \%$ after 2 hours and $22.8 \%$ after 48 hours. In the management of postlaminectomy syndrome the patients need individualized approaches. Further multicentric clinical trials are needed to generalize the results in these patients.
\end{abstract}

Keywords: Laminectomy, Syndrome, Pain, Electrical, Stimulation

\section{Introduction}

Post-laminectomy syndrome specifically refers to pain associated with symptoms not relieved following laminectomy. However, the term often is used more broadly to describe poor outcomes following any type of spinal surgery (1). The classic term laminectomy often is used to describe a partial laminotomy, which is removal of only a portion of the lamina to provide access to a disc herniation. The most frequent surgery preceding a diagnosis of post-laminectomy syndrome is lumbar discectomy (2). Spinal surgery may be performed in patients who do not improve with nonsurgical (conservative) treatment, even though surgical 
results are less predictable in the absence of correlation between specific clinical findings and imaging study results. In those cases, decompression may fail to provide relief, and the result is postlaminectomy syndrome ( 3 , 4).

The causes of poor results or failed back surgery syndrome, a term inclusive of postlaminectomy syndrome and persistent pain after other spinal procedures, including spinal fusion, include poor patient selection (i.e., operating when nerve compression did not exist), nerve root injury at the time of surgery, delayed surgery, infection in the disc space or epidural space, unrecognized lateral spinal stenosis or instability, arachnoiditis, and reherniation. Rarely, the first surgery will have been performed on the wrong side or at the wrong level (malpractice). Surgery may have been performed appropriately and the pathology corrected; however, psychosocial factors may contribute to chronic pain that develops after surgery $(2,3)$.

Transcutaneous electrical nerve stimulation (TENS) is a non-invasive therapeutic modality that was added, more than 30 years ago to existing physical agents used in medicine and physiotherapy for the management of LBP $(5,6)$. TENS units stimulate peripheral nerves via skin surface electrodes at well tolerated intensities and are capable of being self-administered (7).

The development and application of TENS was based on the "Gate Control Theory" conceptualized by Melzack and Wall (8).

The clinical benefit provided by TENS remains controversial. According to the clinical practice guideline developed by the Philadelphia Panel (9), there is poor evidence to support either including or excluding TENS as an intervention for chronic LBP. An earlier systematic review by Van Tulder and colleagues found conflicting results from randomized control trials, concluding that there was no evidence of benefit of TENS for individuals with chronic LBP (10). In contrast, the much earlier Québec Task Force on Spinal Disorders recommended TENS as a rehabilitation modality for symptomatic pain relief, although, in their recommendation, the aim of this systematic review was to determine the effectiveness of TENS in the management of postlaminectomy syndrome (11).

\section{Materials and methods}

From September 2008 till September 2009, all of the patients with postlaminectomy syndrome after lumbar discectomy who were referred to the pain clinic due to failure of conservative treatment including the use of non steroidal anti-inflammatory drugs, different analgesics, anti-depressive, steroid medications, physiotherapy and hydrotherapy were determined. All of the cases were examined by the neurosurgical team and in all of them lumbosacral MRI w/o gadolinium injection performed to detect cases with recurrence of disc herniation or incomplete neural decompression.

For all the cases dynamic radiographies of the lumbosacral spine performed to rule out instability of the spinal anatomy. The patients with recurrence of disc herniation, compressive neural canal or foraminal stenosis or unstable spinal structures were omitted. Our inclusion criteria were as follows: Stable chronic low back pain or stable chronic lumbo-radicular pain in patients with a pain score according to VAS (Visual Analog Scale) equal or more than 4 and patients followed in pain clinics who accepted and signed the informed consent. Other exclusion criteria were: prior ambulatory TENS practice, metabolic lumbo-radicular pathology, surgery within 3 months before inclusion, acute low back pain or bilateral lumbo-radiculalgia, acute radiculalgia, surgery planned within 6 months, pacemaker and symptomatic low back pain. In the selected patients the age, sex, duration of illness following their laminectomy, signs and symptoms and the level of disc herniation were recorded (12). In all the selected cases transcutaneous electrical nerve stimulation performed and the degree of pain were recorded according to VAS before TENS and the after 2 and 48 hours afterwards. Our indicator of successful treatment was reduction of pain score at least $50 \%$ compared to the score before TENS. The protocol of TENS in our patient was conventional with frequency of $40-150 \mathrm{~Hz}$ and the applied amplitude was 50-100 $\mu$ with low intensity according to the patient tolerance. To determine the effectiveness of treatment we used $\mathrm{Z}$ statistical analysis and to determine the role of intervening factors we used $\chi^{2}$ analysis.

\section{Results}

This study is based on the clinical data of 33 patients selected from 85 cases with postlaminectomy syndrome referred to the pain clinic from September 2008 to September 2009, according to the inclusion criteria. 14 cases $(40 \%)$ were female and $21(60 \%)$ were male. The male to female ratio was 1.5 . The average age of our patients was $49.5 \pm 12.5$ years (range: $24-65$ years). Mean duration of postlaminectomy pain was $4.7 \pm 1.3$ years.

The most common location of spinal lesion was L4-5 in 14 cases (40\%). In 10 cases the impaired level was L5-S1 (20\%), in 4 cases at the level of L3-4 (20\%) and in the rest 10\% at other levels. 
In all our cases the pain score was determined using VAS. Before TENS the average pain score was $7 \pm 2$. After performance of transcutaneous nerve stimulation the pain score was recorded in each case. After 2 hours of the mean pain score was $5 \pm 3$. The patients discharged from the Hospital and followed up after 48 hours. In the follow- up exam after 48 hours from TENS, the mean pain score was $4 \pm 2$. The success criteria defined as more than $50 \%$ decrease in the severity of pain. According to this point the successful transcutaneous nerve stimulation occurred in $17.14 \%$ after 2 hours and $22.8 \%$ after 48 hours. We also assessed the statistical relevance of intervening factors with the success of treatment but we found no statistically related intervening factor (Table 1). Complication occurred in only one case as skin redness and slight pain the site of TENS which improved completely with analgesics.

\section{Discussion}

TENS is a non-invasive, very safe method to reduce pain, both acute and chronic. While controversy exists as to its effectiveness in the treatment of chronic pain, a number of systematic reviews or meta-analyses have confirmed its effectiveness for postoperative pain, osteoarthritis, and chronic musculoskeletal pain (13). Conversely, results from the Bone and Joint Decade 2000 - 2010 Task Force on Neck Pain accumulated data showing no clinically significant benefit to TENS for the treatment of neck pain when compared to sham treatment (14). Recent clinical studies and meta-analysis suggest that using adequate intensity of stimulation is necessary to obtain analgesia with TENS. Basic science studies show that high and low frequency TENS produce their effects by activation of opioid receptors in the central nervous system. Specifically, high frequency TENS activates delta-opioid receptors both in the spinal cord and supraspinally (in the medulla) while low frequency TENS activates mu-opioid receptors both in the spinal cord and supraspinally (15). Further high frequency TENS reduces excitation of central neurons that transmit nociceptive information, reduces release of excitatory neurotransmitters (glutamate) and increases the release of inhibitory neurotransmitters (GABA) in the spinal cord, and activates muscarinic receptors centrally to produce analgesia (in effect, temporarily blocking the pain gate). Low frequency TENS also releases serotonin and activates serotonin receptors in the spinal cord, releases GABA, and activates muscarinic receptors to reduce excitability of nociceptive neurons in the spinal cord (16).The development and application of TENS was based on the Gate Control Theory conceptualized by Melzack and Wall (8) According to this theory, the stimulation of large diameter, (A-beta) primary sensory afferents activates inhibitory interneurons in the substantia gelatinosa of the spinal cord dorsal horn and, thereby, attenuates the transmission of nociceptive signals from small diameter A-delta and C fibers (8). Supraspinal mechanisms involving the endogenous opioid system have also been described (17).

Several types of TENS applications, differing in frequency, amplitude, pulse width and waveform, are used in clinical practice. The two most common application modes include: 1) high frequency or conventional TENS (40 to $150 \mathrm{~Hz}, 50$ to 100 millisecond pulse width, low intensity), and 2) low frequency or so called acupuncture-like TENS ( 1 to $4 \mathrm{~Hz}, 100$ to 400 uses pulse width, high intensity) (17). Conventional TENS is associated with a faster onset and shorter duration of analgesia compared to acupuncture-like TENS (18). However, whether there is a significant difference in clinical effectiveness between high frequency and low frequency modes is unclear and not well defined (19). Indeed, patient preference for, and response to, different stimulation settings may be highly individualized (20). Three other standard modes of TENS include: 1) Brief-Intense TENS (greater than $80 \mathrm{~Hz}$, greater than 150 millisecond pulse width, comfortable-tolerable intensity), 2) Burst TENS (bursts of pulses delivered at a low frequency of less than $10 \mathrm{~Hz}$ and at a comfortable intensity) and 3)Modulation TENS (one or more stimulation parameters are randomly modulated during therapy). Adverse reactions reported with TENS include skin irritation at the site of electrode placement. TENS is contraindicated in patients with cardiac pacemakers due to the potential of interfering with pacemaker activity (21).

The clinical benefit provided by TENS remains controversial. In a systematic review, Flowerdew and co-workers, found limited evidence for the usefulness of TENS in individuals with chronic LBP, citing the need for more definitive, high quality randomized-controlled studies (22).

The results of trial of Cheing and colleagues showed a significant decrease in subjective pain intensity with active TENS treatment compared to placebo. Pain reduction was progressive over the course of the 60-minute treatment session and then quickly reached a plateau once stimulation ended. The pain reduction seen at the end of stimulation was maintained for the entire 60-minute post-treatment time interval assessed (23).

In comparison, the results of the second trial by Deyo demonstrated no significant differences between active TENS and placebo for any of the outcomes measured, including pain, functional status, range of motion, and use of medical services. In a third of the participants, minor skin irritation occurred at the site of electrode placement. These adverse effects were observed equally in the active TENS and placebo groups. One participant 
randomized to placebo TENS developed severe dermatitis four days after beginning therapy and was required to withdraw (24).

In our study we had the similar complication presented as mild redness in one of our cases.

\section{Conclusions}

Pain is a multidimensional experience that has both peripheral and central substrates (25). Peripheral nociceptor sensitization, dorsal receptor field expansion, and altered central neuromodulation of pain may all play a role in the transition from acute to chronic LBP. It is thought that the neuroregulatory peripheral and central effects of TENS may be more effective with higher intensity applications such as acupuncture-like TENS. The possibility exists that individualized benefits from TENS cannot be precisely captured with small randomized controlled trials. Indeed, individual response to different stimulation settings (frequency, amplitude, pulse width, waveform) may be quite variable $(13,20)$. Furthermore, particular subgroups with chronic LBP may respond to TENS better than others. LBP has multiple etiologies (26). Refining the definition and classification of chronic LBP and clarifying its underlying pathophysiological mechanisms will help to promote more uniform study populations. While studying the effects of short term TENS therapy is important, understanding the risks and benefits of long-term use is probably more relevant in the management of post laminectomy syndrome.

\section{References}

American Physical Therapy Association. (1993). Electrical Stimulation: Management of Pain. American Physical Therapy Association Anthology, Vol. 2, 198-212.

Barr JO. (1999). Transcutaneous Electrical Nerve Stimulation for pain management. In: Nelson RM, Hayes KW, Currier DP editor(s). Clinical electrotherapy. $3^{\text {rd }}$ Edition. Appleton \& Lange, 291-354.

Belanger AY. (2002). Evidence based guide to therapeutic physical agents. Lippincott Williams \& Wilkins.

Bjordal JM, Johnson MI, Ljunggreen AE. (2003). Transcutaneous electrical nerve stimulation (TENS) can reduce postoperative analgesic consumption. A metaanalysis with assessment of optimal treatment parameters for postoperative pain. Eur J Pain, 7 (2). 181-8.

Cheing GL. Hui-Chan CW. (1999). Transcutaneous electrical nerve stimulation: nonparallel antinociceptive effects on chronic clinical pain and acute experimental pain. Archives of Physical Medicine \& Rehabilitation, 80(3):305-12.

Deyo RA, Wash NE, Schoenfeld LS, Ramamurthy S. (1990). Can trials of physical treatments be blinded: the example of transcutaneous electrical nerve stimulation for chronic pain. American Journal of Physical Medicine and Rehabilitation, 69:6-10.

Deyo RA, Weinstein JN. (2001). Low back pain. New England Journal of Medicine, 344(5):363-70.

Epstein, N. E. (2004). Lumbar Laminectomy for the Resection of Synovial Cysts and Coexisting Lumbar Spinal Stenosis or Degenerative Spondylolisthesis: An Outcome Study. Spine Vol 29, 9: 1049-1055.

Flowerdew MW, Gadsby JG. (1997). A review of the treatment of chronic low back pain with acupuncture-like transcutaneous electrical stimulation and transcutaneous electrical nerve stimulation. Complemen tary Therapies in Medicine, 5:193-201.

Heirhoff KB, Button CV. (1985). The evaluation of the failed back surgery syndrome. Orthop Clin Norch Am, 16: 417-444.

Jette AM, Delitto A. (1997). Physical therapy treatment choices for musculoskeletal impairments. Physical Therapy, 77:145-54.

Johnson M, Martinson, M. (2006). Efficacy of electrical nerve stimulation for chronic musculoskeletal pain: A meta-analysis of randomized controlled trials. Pain, 130 (1): 157-165.

JohnsonMI, Ashton CH, Thompson JW. (1991). An in-depth study of longterm users of transcutaneous electrical nerve stimulation (TENS). Implications for clinical use of TENS, 44(3), 221-9.

Kalra A, Urban MO, Sluka KA. (2001). Blockade of opioid receptors in rostral ventral medulla prevents antihyperalgesia produced by transcutaneous electrical stimulation (TENS). JPET, 298:257-63.

Linacre J.M. Visual Analog Scale. (1998). Rasch Measurement Transactions, 12:2: 639.

Melzack R. (1999). From the gate to the neuromatrix. Pain, 6 (Suppl), 121-6. 
Philadelphia Panel. (2001). Philadelphia Panel evidence-based clinical practice guidelines on selected rehabilitation interventions for low back pain. Phys Ther., 81(10), 1641-74.

Québec Task Force on Spinal Disorders. (1987). Scientific approach to the assessment andmanagement of activity-related spinal disorder: a monograph for clinicians. Spine, 12, 51-9.

Rakel B, Frantz R. (2003). Effectiveness of transcutaneous electrical nerve stimulation on postoperative pain with movement. J Pain, 4 (8). 455-64.

Rushton DN. (2002). Electrical stimulation in the treatment of pain. Disability and Rehabilitation, 2 (8), 407-15.

Sierpina VS, Curtis P, Doering J. (2002). An integrative approach to low back pain. Clinics in Family Practice, 4(4); 817-31.

Sluka KA, Walsh D. (2003). Transcutaneous electrical nerve stimulation basic science mechanisms and clinical effectiveness. Journal of Pain, Vol 4, (3), 109- 21.

Soin, A. (2008). Functional Outcomes in Patients with Chronic Nonmalignant Pain on Long-term Opiod Therapy. Pain Practice, Vol 8, 5: 379-384.

The Bone and Joint Decade. (2000-2010). Task Force on Neck Pain and Its Associated Disorders: Executive Summary. Spine 33 (4S Supplement), (2008): 5-7.

Van Tulder MW, Koes BW, Bart W, Bouter LM. (1997). Conservative Treatment of Acute and Chronic Nonspecific Low Back Pain: A systematic review of the most common interventions. Spine: 22(18), 2128-56.

Waddell G. (1987). Clinical assessment of lumbar Impairment. Clin Orthop, 221: 110- 120. 